\title{
Hydrodynamic Analysis of 3-SPS Wave Energy Conversion Device
}

\author{
Zhifei Ji ${ }^{1,2,3}$, Xiaodong Yuan ${ }^{1}$, Min $\operatorname{Lin}^{1 *}$ and Jianyu Fan ${ }^{1}$ \\ ${ }^{1}$ College of Ocean Equipment and Mechanical Engineering, Jimei University, Xiamen 361021, Fujian Province, China \\ ${ }^{2}$ Fujian Province Key Laboratory of Ocean Renewable Energy Equipment, Xiamen, Fujian, 361021, China \\ ${ }^{3}$ School of Electro-Mechanical Engineering, Xidian University, Xi'an 710071, Shaanxi Province, China
}

\begin{abstract}
Wave energy has the advantages of high energy density, renewability, and wide distribution, and has been highly valued by many coastal countries. The wave energy conversion device can convert wave energy into electric energy, which is of great significance for alleviating problems such as energy crisis and greenhouse effect. The traditional wave energy conversion device can only gain the energy along the heave direction, and the kinetic energy of the buoy is not fully utilized. To improve the energy utilization efficiency of the wave energy conversion device, this paper proposed a new type of 3-SPS wave energy conversion device. Based on linear waves and Lagrangian equation, a hydrodynamic model of the device was established. The displacement and velocity of the device float under the action of linear waves were analyzed. The results show that the 3-SPS wave energy conversion device can collect the kinetic energy of the buoy in its heaving, surging and pitching movement at the same time; the kinetic energy of the buoy in the heaving direction is much greater than the kinetic energy in the surging and pitching directions; the buoy can capture kinetic energy in multiple directions of motion, indicating that the 3-SPS wave energy conversion device has a high energy utilization efficiency. This paper provides some useful references for the optimal design of the new wave energy device.
\end{abstract}

\section{Introduction}

As a kind of clean energy, wave energy has the characteristics of large reserves, high energy flow density, wide distribution, and easy conversion of energy forms [1], and has been highly valued by many coastal countries. In December 2016, the "13th Five-Year Plan for Energy Technology Innovation" issued by the National Energy Administration of China pointed out that research on high conversion rate wave energy power generation technology and research and development of wave energy power generation devices is one of the important tasks of energy technology innovation planning. China is a large maritime country with a total coastline of more than 32,000 kilometers, and the usable wave energy is very abundant [2-3]. Therefore, research on wave energy power generation technology is of great significance for ensuring energy security, maintaining sustainable economic and social development, and enhancing the international competitiveness of marine technology in China.

The wave energy conversion device can realize the conversion of wave energy to electric energy [4-6]. The buoy-type wave energy conversion device is one of the research hotspots in the field of wave energy power generation technology [7-9]. Under the action of waves, the buoy produces motion in 6 directions, namely: yawing, heaving, pitching, swaying, rolling and surging. The traditional single-degree-of-freedom wave energy conversion device only collects the energy of the buoy along the heaving direction, and the kinetic energy of the buoy in other directions is not fully utilized, which limits the energy capture efficiency of the single-degree-offreedom wave energy conversion device to a certain extent. Traditional methods to improve the energy capture efficiency of the device often focus on how to optimize the shape and size of the buoy, and ignore the influence of the freedom of the device on the energy capture efficiency. For this reason, this paper proposed a new type of 3-SPS wave energy conversion device, which can collect the energy in the heaving, surging and pitching directions at the same time. The hydrodynamic model of the device was established thought the Lagrangian method. Based on the Runge-Kutta method, the hydrodynamics of the device was simulated, and the kinematic characteristics of the device were obtained. Finally, the energy utilization efficiency of the device was analyzed.

\section{Wave model}

Wave models mainly include linear waves, nonlinear waves and random waves. This paper mainly studied the hydrodynamic characteristics of 3-SPS wave energy conversion under the action of linear waves. Linear wave refers to a wave with a sinusoidal profile [164], and its schematic diagram is shown in Figure 1.

* Corresponding author: 201761000009@jmu.edu.cn 


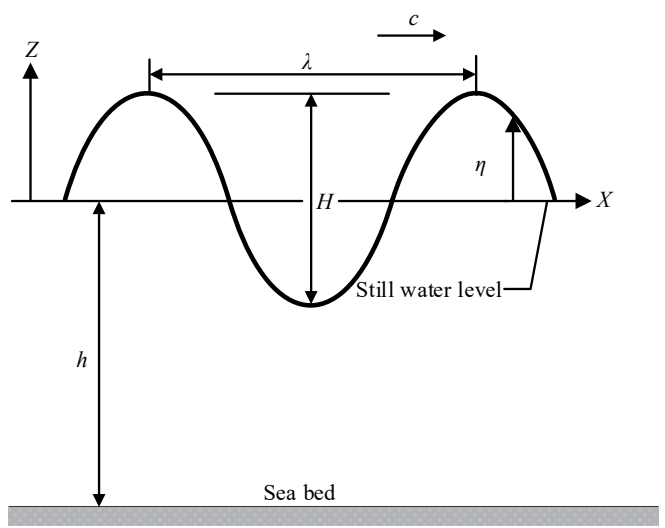

Figure 1. Linear wave

The parameters of the linear wave shown in Figure 1 are as follows:

$h$ : Water depth, refers to the distance from the bottom of the water to the surface of still water.

$H$ : Wave height, refers to the depth from the crest to the trough.

$\lambda$ : Wavelength, refers to the distance between two adjacent wave crests.

$\eta$ : Vertical free surface displacement, which is a function of displacement $x$ in the direction $X$ and $t$.

$c$ : Wave speed.

\subsection{Wave dynamics model}

The motion of the water mass points that make up the linear wave can be described by its velocity potential. Assuming that the flow below the free surface of water is a non-rotational fluid, its velocity potential is:

$$
\mathbf{V}=\nabla \phi
$$

For the stable incompressible fluid, there is:

$$
\nabla^{2} \phi=0
$$

Equation (2) is an elliptic partial differential equation, which can be solved by applying boundary conditions (free surface condition of motion, seabed condition and dynamic free surface condition), and the velocity potential $\phi$ can be obtained as follows:

$$
\phi^{ \pm}= \pm \frac{H}{2} \frac{g}{k c} \frac{\cosh [k(z+h)]}{\cosh (k h)} \sin [k(x \mp c t)]
$$

$\phi^{ \pm}$takes "+" to indicate right-traveling waves and "-." to indicate left-traveling waves. In this paper, we assume that the linear wave studied is a right-traveling wave, and the free surface displacement is:

$$
\eta=\frac{H}{2} \cos [k(x-c t)]
$$

The wave number $k$ is as follows:

$$
k=\frac{2 \pi}{\lambda}
$$

The time difference between two consecutive peaks is the period $T$ of the linear wave. From equation (5), we can know that:

$$
k c=\frac{2 \pi}{T}=2 \pi f \equiv \omega
$$

In the equation, $f$ is the frequency of the wave in Hz. $\omega$ is the angular frequency in $\mathrm{rad} / \mathrm{s}$. From equations (5) and (6), the wave velocity can be obtained as:

$$
c=\frac{\lambda}{T}
$$

Therefore, the velocity potential can be expressed as a function of angular frequency:

$$
\phi=\frac{H}{2} \frac{g}{\omega} \frac{\cosh [k(z+h)]}{\cosh (k h)} \sin [k x-\omega t]
$$

In this case, the wavelength can be expressed as:

$$
\lambda=\frac{2 \pi}{k}=\frac{g T^{2}}{2 \pi} \tanh \left(\frac{2 \pi h}{\lambda}\right)=c T
$$

The propagation characteristics of linear waves and the movement of water quality points can be analyzed by equations (4)-(9).

\subsection{Wave energy model}

From the previous research [165], the energy and power of the linear wave can be obtained as:

$$
\begin{gathered}
E=\frac{\rho g^{3 / 2} H^{2} h^{1 / 2} T b}{8} \\
\mathbf{P}=\rho b \frac{1}{T} \int_{0}^{T} \int_{-h}^{0} \frac{\partial \phi}{\partial t} \nabla \phi d z d t \\
=\frac{\rho g H^{2} c b}{16}\left[\frac{2 k h}{\sinh (2 k h)}+1\right] \mathbf{i}
\end{gathered}
$$

\section{Dynamic model of 3-SPS wave energy conversion device}

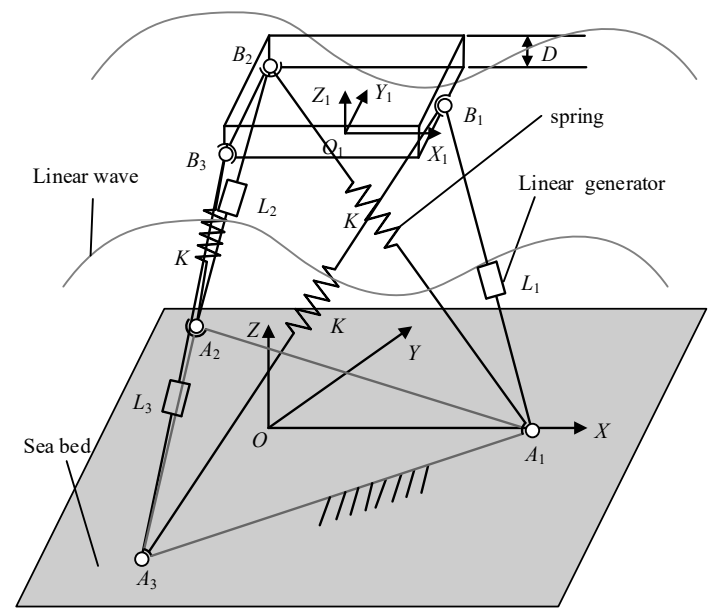

Figure 2. 3-SPS wave energy conversion device

The 3-SPS wave energy conversion device proposed in this paper is shown in Figure 2. The device consists of a buoy, four linear generators connected with the buoy, and a spring. The length and width of the buoy are both $L$ and the height is $D$. The lower platform $\mathrm{A}_{1} \mathrm{~A}_{2} \mathrm{~A}_{3}$ is fixed to the seabed by a spherical pair, and its shape is a regular triangle with a side length of $L$. The spring coefficients of springs $\mathrm{A}_{1} \mathrm{~B}_{2}, \mathrm{~A}_{2} \mathrm{~B}_{3}$, and $\mathrm{A}_{3} \mathrm{~B}_{1}$ are $K$, and their original lengths are $L_{01}, L_{02}$, and $L_{03}$, respectively. The origin of the static coordinate system is fixed at the center of the lower 
platform, and the direction of the coordinate axis is shown in Figure 2. The dynamic model of the energy conversion device is established based on the Lagrangian method. The general form of the equation is:

$$
\frac{d}{d t} \frac{\partial T_{K}}{\partial \dot{\mathbf{q}}}-\frac{\partial T_{K}}{\partial \mathbf{q}}+\frac{\partial U}{\partial \mathbf{q}}=\mathbf{Q}_{k}
$$

In the equation, $T_{K}$ is the total kinetic energy of the system; $U$ is the total potential energy of the system; $\boldsymbol{Q}$ is the non-conservative generalized force; $\boldsymbol{q}$ is the generalized coordinate.

Assuming that the spring is linear elastic and the mass is zero, the propagation direction of the wave is assumed to be the positive direction of the $X$ axis. Under the action of the linear wave, the main form of movement of the buoy is the movement along the $X$ axis, the movement along the $Z$ axis and the rotation around the $Y$ axis. Therefore, three generalized coordinates should be used to describe the movement of the buoy.

$$
\mathbf{q}=\left[\begin{array}{lll}
q_{1} & q_{2} & q_{3}
\end{array}\right]^{T}=\left[\begin{array}{lll}
x & z & \beta
\end{array}\right]^{T}
$$

Assuming that the mass of the linear motor is small, the kinetic energy of the system is mainly produced by the buoy. The kinetic energy of the buoy includes translational kinetic energy and rotational kinetic energy, and its expression is as follows:

$$
T_{K}=\frac{1}{2} \dot{\mathbf{q}}^{\mathrm{T}} \mathbf{M} \dot{\mathbf{q}}
$$

In the equation,

$$
\mathbf{M}=\left[\begin{array}{ccc}
m+a_{w y} & 0 & 0 \\
0 & m+a_{w y} & 0 \\
0 & 0 & I_{y}+A_{w}
\end{array}\right]
$$

The potential energy of the system is:

$$
U=\frac{1}{2} \rho g A_{w p} q_{2}^{2}+\frac{1}{2} C q_{3}^{2}+\frac{1}{2} \sum_{i=1}^{3} K_{i}\left(L_{i}-L_{i 0}\right)^{2}
$$

Considering the fluid damping, the non-conservative generalized force on the system is:

$$
\mathbf{Q}_{k}=\left[\begin{array}{c}
-b_{v y} \dot{q}_{1} \\
F_{z 0} \cos (\omega t)-\left(b_{r z}+b_{v z}\right) \dot{q}_{2} \\
M_{\theta 0} \cos (\omega t)-b_{r \theta} \dot{q}_{3}
\end{array}\right]
$$

Substituting equations (15), (16) and (17) into equation (12), the dynamic model of the system can be obtained as:

$$
\mathbf{M} \ddot{\mathbf{q}}+\mathbf{B} \cdot \dot{\mathbf{q}}+\mathbf{G}=\mathbf{F}
$$

In the equation,

$$
\begin{aligned}
& \mathbf{M}=\left[\begin{array}{ccc}
m+a_{\omega y} & 0 & 0 \\
0 & m+a_{\omega z} & 0 \\
0 & 0 & I_{x}+A_{\omega}
\end{array}\right] \\
& \mathbf{G}=\left[\begin{array}{lll}
\frac{\partial U}{\partial q_{1}} & \frac{\partial U}{\partial q_{2}} & \frac{\partial U}{\partial q_{3}}
\end{array}\right]^{T} \\
& \mathbf{B}=\left[\begin{array}{ccc}
b_{v y} & 0 & 0 \\
0 & b_{r z}+b_{v z}+b_{p z} & 0 \\
0 & 0 & b_{r \theta}
\end{array}\right] \\
& \mathbf{F}=\left[\begin{array}{lll}
0 & F_{z o} \cos \omega t & M_{\theta 0} \sin \omega t
\end{array}\right]^{T}
\end{aligned}
$$

The elements in matrix $\mathbf{G}$ are as follows:

$$
\begin{aligned}
& G_{1}=\frac{\partial U}{\partial q_{1}}=2 K\left\{1-L_{0}\left[\left(q_{1}-\frac{L \cos q_{3}}{2}-\frac{D \sin q_{3}}{2}+\frac{L}{2}\right)^{2}+\right.\right. \\
& \left.\left.\left(q_{2}-\frac{D \cos q_{3}}{2}+\frac{L \sin q_{3}}{2}\right)^{2}\right]^{-\frac{1}{2}}\right\} \text {. } \\
& \left(q_{1}-\frac{L \cos q_{3}}{2}-\frac{D \sin q_{3}}{2}+\frac{L}{2}\right)+ \\
& 2 K\left(q_{1}+\frac{L \cos q_{3}}{2}-\frac{D \sin q_{3}}{2}-\frac{L}{2}\right) . \\
& \left\{1-L_{0}\left[\left(q_{1}+\frac{L \cos q_{3}}{2}-\frac{D \sin q_{3}}{2}-\frac{L}{2}\right)^{2}+\right.\right. \\
& \left.\left.\left(q_{2}-\frac{D \cos q_{3}}{2}-\frac{L \sin q_{3}}{2}\right)^{2}\right]^{-\frac{1}{2}}\right\} \\
& G_{2}=\frac{\partial U}{\partial q_{2}}=\rho g A_{w p} q_{2}+2 K\left(q_{2}-\frac{D \cos q_{3}}{2}+\frac{L \sin q_{3}}{2}\right) . \\
& \left\{1-L_{0}\left[\left(q_{1}-\frac{L \cos q_{3}}{2}-\frac{D \sin q_{3}}{2}+\frac{L}{2}\right)^{2}+\right.\right. \\
& \left.\left.\left(q_{2}-\frac{D \cos q_{3}}{2}+\frac{L \sin q_{3}}{2}\right)^{2}\right]^{-\frac{1}{2}}\right\}+ \\
& 2 K\left\{1-L_{0}\left[\left(q_{1}+\frac{L \cos q_{3}}{2}-\frac{D \sin q_{3}}{2}-\frac{L}{2}\right)^{2}+\right.\right. \\
& \left.\left.\left(q_{2}-\frac{D \cos q_{3}}{2}-\frac{L \sin q_{3}}{2}\right)^{2}\right]^{-\frac{1}{2}}\right\} \text {. } \\
& \left(q_{2}-\frac{D \cos q_{3}}{2}-\frac{L \sin q_{3}}{2}\right) \\
& G_{3}=C q_{3}+2 K\left\{1-L_{0}\left[\left(q_{1}-\frac{L \cos q_{3}}{2}-\frac{D \sin q_{3}}{2}+\frac{L}{2}\right)^{2}+\right.\right. \\
& \left.\left.\left(q_{2}-\frac{D \cos q_{3}}{2}+\frac{L \sin q_{3}}{2}\right)^{2}\right]^{-\frac{1}{2}}\right\} \text {. } \\
& {\left[\left(q_{1}-\frac{L \cos q_{3}}{2}-\frac{D \sin q_{3}}{2}+\frac{L}{2}\right)\left(\frac{L \sin q_{3}}{2}-\frac{D \cos q_{3}}{2}\right)+\right.} \\
& \left.\left(q_{2}-\frac{D \cos q_{3}}{2}+\frac{L \sin q_{3}}{2}\right)\left(\frac{D \sin q_{3}}{2}+\frac{L \cos q_{3}}{2}\right)\right]+ \\
& 2 K\left\{1-L_{0}\left[\left(q_{1}+\frac{L \cos q_{3}}{2}-\frac{D \sin q_{3}}{2}-\frac{L}{2}\right)^{2}+\right.\right. \\
& \left.\left.\left(q_{2}-\frac{D \cos q_{3}}{2}-\frac{L \sin q_{3}}{2}\right)\right]^{-\frac{1}{2}}\right\} \text {. } \\
& {\left[-\left(q_{1}+\frac{L \cos q_{3}}{2}-\frac{D \sin q_{3}}{2}-\frac{L}{2}\right)\left(\frac{L \sin q_{3}}{2}+\frac{D \cos q_{3}}{2}\right)+\right.} \\
& \left.\left(q_{2}-\frac{D \cos q_{3}}{2}-\frac{L \sin q_{3}}{2}\right)\left(\frac{D \sin q_{3}}{2}-\frac{L \cos q_{3}}{2}\right)\right]
\end{aligned}
$$




\section{Numerical examples}

\subsection{Wavelength solution steps}

Equation (18) describes the dynamic model of the 3-SPS wave energy conversion device. It can be seen that, to analyze its dynamic characteristics, the parameters of the wave must first be calculated. The fixed-point iteration method is used to calculate the wavelength of linear waves. The main steps are as follows:

Step 1: Given the accuracy $\varepsilon$, the linear wave period $T$ and the distance from the static water surface to the seabed $h$.

Step 2: Given the initial iteration value $\lambda^{(0)}$ of wavelength.

Step3: Calculate $F_{A}(\lambda)=\lambda$, and $F_{B}(\lambda)=\frac{g T^{2}}{2 \pi} \tanh \left(\frac{2 \pi h}{\lambda}\right)$

Step 4: If $\left|F_{A}(\lambda)-F_{B}(\lambda)\right| \geq \varepsilon$ set $\lambda=F_{B}(\lambda)$, repeat step 3.

Step 5: If $\left|F_{A}(\lambda)-F_{B}(\lambda)\right| \leq \varepsilon$, output $\lambda$.

Step 6: End.

\subsection{Hydrodynamic analysis of 3-SPS wave energy conversion device}

Supposing the wave height $H$ is $0.2 \mathrm{~m}$, the period $T$ is $6 \mathrm{~s}$, and the water depth is $100 \mathrm{~m}$. The size of the buoy is selected as: $L=1 \mathrm{~m}, D=0.1 \mathrm{~m}, d=0.05 \mathrm{~m}$. The parameters of the spring are: $L_{0}=4 \mathrm{~m}, K=10 \mathrm{~m}$. First, we calculated the value of each coefficient in equation (18).

Table 1. Values of the coefficients in formula (18)

\begin{tabular}{ccc}
\hline Coefficient & Value & Unit \\
\hline $\mathrm{m}$ & 51.50 & $\mathrm{~kg}$ \\
$a_{\mathrm{wy}}$ & 2.02 & $\mathrm{~kg}$ \\
$a_{\mathrm{wz}}$ & 454.19 & $\mathrm{~kg}$ \\
$A_{w}$ & 15.03 & $\mathrm{~kg} \cdot \mathrm{m}^{2}$ \\
$I_{x}$ & 4.33 & $\mathrm{Kg} \cdot \mathrm{m}^{2}$ \\
$C$ & 849.58 & $\mathrm{~N} \cdot \mathrm{m} / \mathrm{rad}$ \\
$b_{\mathrm{rz}}$ & 1065.50 & $\mathrm{~N} \cdot \mathrm{s} / \mathrm{m}$ \\
$b_{r \theta}$ & 88.79 & $\mathrm{~N} \cdot \mathrm{m} \cdot \mathrm{s} / \mathrm{rad}$ \\
$b_{v y}$ & 114.91 & $\mathrm{~N} \cdot \mathrm{s} / \mathrm{m}$ \\
$b_{v z}$ & 114.91 & $\mathrm{~N} \cdot \mathrm{s} / \mathrm{m}$ \\
$b_{\mathrm{pz}}$ & 0 & $\mathrm{~N} \cdot \mathrm{s} / \mathrm{m}$ \\
$A_{\mathrm{wp}}$ & 1 & $\mathrm{M}$ \\
$F_{\mathrm{z} 0}$ & 2012.10 & $\mathrm{~N}$ \\
$M_{\theta 0}$ & 18.77 & $\mathrm{~N} \cdot \mathrm{m}$ \\
$a_{\mathrm{z}}$ & 0 & $\mathrm{rad}$ \\
\hline
\end{tabular}

Solving equation (18) based on Runge-Kutta method, the movement rules of the moving platform along $X$ axis and $Z$ axis and rotation around $Y$ axis can be obtained, as shown in Figure 3-5.

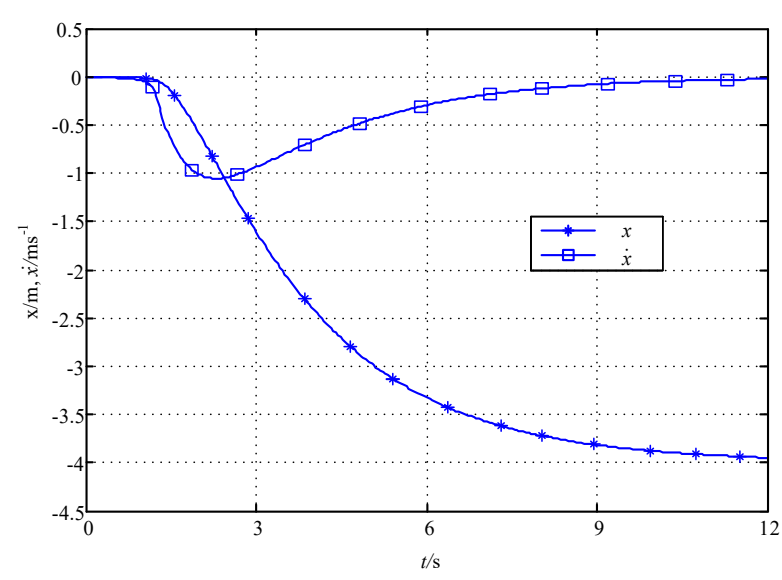

Figure 3. $X$-direction motion law of the moving platform

It can be seen from Figure 3 that under the action of linear waves, the moving platform of the 33-SPS wave energy conversion device has a certain speed in the $X$ direction at the beginning of the movement. When the system is stabilized, the translational kinetic energy in the $X$ direction gradually decreases to zero. For this wave energy conversion device, the wave energy captured by the buoy is mainly converted into the translational kinetic energy of the buoy along the $Z$ direction and the rotational kinetic energy of the buoy around the $Y$ axis. The instantaneous power of the 3-SPS wave energy conversion device is shown in Figure 6.

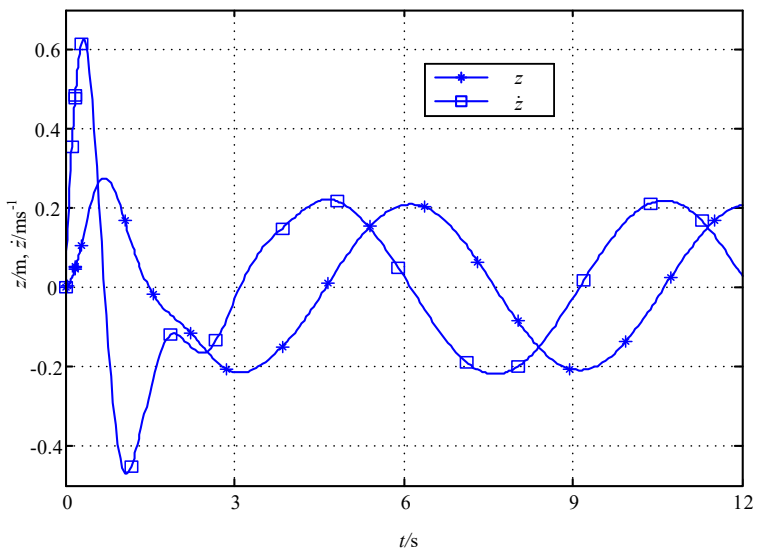

Figure 4 Z-direction motion law of the moving platform

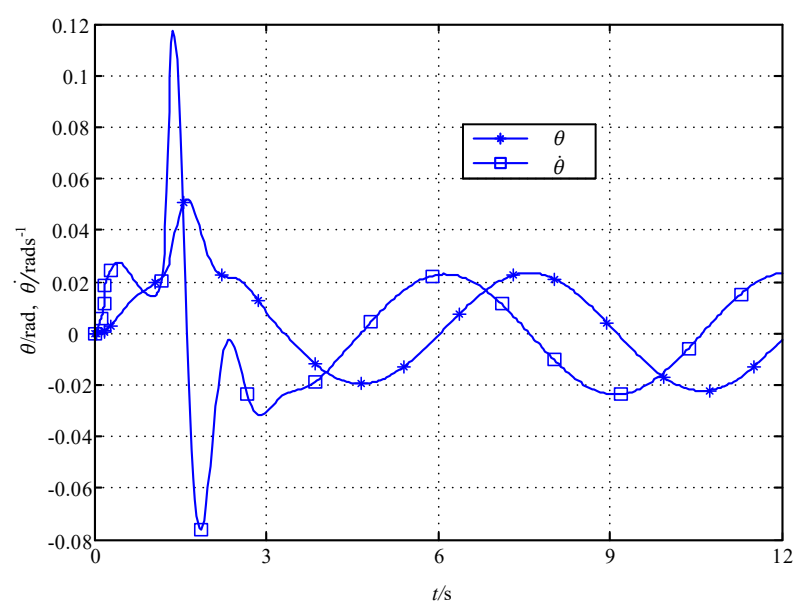

Figure $5 Y$-direction rotation law of the moving platform 


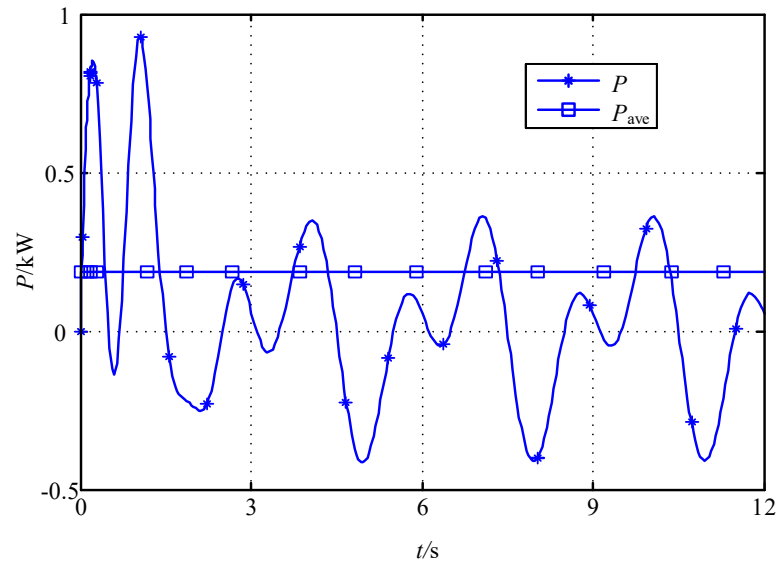

Figure 6 The generating power of 3-SPS wave energy conversion device

It can be seen from Figure 6 that the power generation of the 3-SPS wave energy conversion device is $0.186 \mathrm{~kW}$. Based on equation (11), the linear wave power is $0.236 \mathrm{~kW}$, and the power generation efficiency of the 3-SPS wave energy conversion device is $78.76 \%$.

\section{Conclusion}

This paper proposed a new type of 3-SPS wave energy conversion device. First, a linear wave kinematics model was derived. On this basis, a hydrodynamic model of the 3-SPS wave energy conversion device under the action of linear waves was established. This hydrodynamic model needs to consider the mutual coupling between rigid body dynamics and fluid dynamics. Based on the Runge-Kutta method, the hydrodynamic model of the 3-SPS wave energy conversion device was solved, and the utilization rate of the wave energy by the system was analyzed. The research results show that under the action of linear waves, the moving platform of the 3-SPS energy harvester has a certain speed along the surging direction at the initial stage of movement. When the system is stable, the translational kinetic energy in the surging direction gradually decays to zero. The wave energy captured by the buoy is mainly transformed into translational kinetic energy in the heaving direction and rotational kinetic energy in the pitching direction of the buoy. The wave energy utilization rate of the 3-SPS wave energy conversion device can reach $78.76 \%$.

\section{Acknowledgments}

The authors gratefully acknowledge the support of the Natural Science Foundation of Fujian Province, China (Grant No. 2018J05088), the Scientific Research Foundation of Jimei University (Grant No. ZQ2017005), Training Program of the National Natural Science Foundation of Jimei University (ZP2020047) and the Young and Middle-aged Teacher Education Scientific Program of Fujian Province (Grant No. JAT200250).

\section{References}

1. F. Mwasilu, J. Jung. Potential power generation from ocean wave renewable energy source: a comprehensive review on state-of-the art technology and future prospects. IET Renewable Power Generation, 13, 3 (2019).

2. D. Zhang, W. Li, Y. G. Lin. Wave energy in China: current status and perspectiv. Renewable Energy, 34, 10 (2009).

3. D. Zhang, J. Wang, Y. Lin, Y. Si, C. Huang, J. Yang, B. Huang. Present situation and future prospect of renewable energy in China. Renewable and sustainable energy reviews, 76 (2017).

4. J. Falnes. A review of wave-energy extraction. Marine Structures, 20, 4 (2007)

5. A. F. Falcão. Wave energy utilization: A review of the technologies. Renewable and sustainable energy reviews, 14 (2010).

6. Y. Lin, J. Bao, H. Liu, W. Li, L. Tu, D. Zhang. Review of hydraulic transmission technologies for wave power generation. Renewable and Sustainable Energy Reviews, 50 (2015).

7. Y. You, W. Li, W. Liu, X. Li, F. Wu. Development status and perspective of marine energy conversion systems. Automation of Electric Power Systems, 34, 14 (2010).

8. Y. Liu, R. Jia, J. Zhang. Research status and prospect of the wave power generation technology. Journal of Ocean Technology, 2016, 35(5): 100-104.

9. L. Iraide, J. Andreu, S. Ceballos. Review of wave energy technologies and the necessary powerequipment. Renewable and Sustainable Energy Reviews, 27 (2013). 